\title{
An X-ray Image Panorama System Using Robust Feature Matching and Perception-Based Image Enhancement
}

\author{
Weiwei Wang ${ }^{\dagger}$, Oubong Gwun ${ }^{+\dagger}$
}

\begin{abstract}
This paper presents an $\mathrm{x}$-ray medical image panorama system which can overcome the smallness of the images that exist on a source computer during remote medical processing. In the system, after the standard medical image format DICOM is converted to the PC standard image format, a MSR algorithm is used to enhance X-ray images of low quality. Then SURF and Multi-band blending are applied to generate a panoramic image. Also, this paper evaluates the proposed SURF based system through the average gray value error and image quality criterion with $\mathrm{X}$-ray image data by comparing with a SIFT based system. The results show that the proposed system is superior to SIFT based system in image quality.
\end{abstract}

Key words: Image Stitching, MSR, SURF, Multi-band Blending, Remote Cooperation, X-ray images

\section{INTRODUCTION}

As X-ray imaging becomes widespread method in medical imaging [1], it has been often applied in an applications such as clinical remote cooperation. During the remote cooperation process, the physicians not only want to gather partial organ information, but also watch the organs at the same time. In the practical process, owing to the restrictions of facilities, remote doctors can see only specified regions.

It is medical common sense that concentration only on local pathologic images may lead to misdiagnosis [2]. To overcome this problem, after having stitched local images into a panoramic im-

* Corresponding Author: Oubong Gwun, Address: (561756) Room 601, No.7 Building, Dept.of Computer Sci. \& Eng.. Chonbuk National University, Jeonju, South-Korea TEL : +82-63-270-3402, FAX : +82-63-270-2394, E-mail : obgwun@chonbuk.ac.kr

Receipt date: Sep. 14, 2011, Revision date: Nov. 23, 2011 Approval date: Mar. 6, 2012

${ }^{+}$Computer Science \& Computer Engineering, Chonbuk National University Computer Graphics Lab (E-mail: lovechewei87@163.com)

${ }^{++}$Computer Science \& Computer Engineering, Chonbuk National University Computer Graphics Lab age which can have more comprehensive information, the panoramic image is saved into a database of the source computer, so that image may be shared through network [3].

The standard format of medical images is DICOM (Digital Image and Communications in Medicare), but the general software in Windows currently cannot display this format directly [4] Therefore our system converts the DICOM format to a standard image file format, then processes and displays them. Meanwhile $\mathrm{X}$-ray images may be unclear, for example, the conditions of the working and operation environment. Thus it needs $\mathrm{x}$-ray image enhancement. MSR (Multi-Scale Retinex) is a perception-based image enhancement algorithm that can provide color constancy and dynamic range compression [5].

In general, stitching contains two main processes, which are an image matching and an image blending. Image matching is used to find mutual relationship between images, which is significant for stitching performance and speed. Traditional methods of image matching are the direct method and feature-based method [6]. The typical feature-based methods are SIFT (Scale Invariant 
Feature Transform) [7] and SURF (Speeded up Robust Features) [8][14]. Because their robustness to different deformation form is depended on applications, this paper will compare the two methods for X-ray images, based on image quality and time cost criterion.

Image blending is another part of image stitching. There are also many methods for image blending; the standard methods are linear blending and multi-band blending. This paper chooses multi-band blending because it can obtain seamless panoramic images easily.

In short, this paper presents a panorama system composed of an MSR image enhancement algorithm, a SURF feature point selecting algorithm, a RANSAC(Random Sample Consensus) outlier correcting algorithm, and a Multi-band blending algorithm for the remote medical cooperation process.

This paper is arranged as follows. Section 2 gives the whole processing overview of the proposed method. Section 3 details the pre-processing and the main processing of the proposed panorama system by comparing SIFT based stitching and SURF based stitching. Section 4 shows the $\mathrm{ex}^{-}$ perimental results of the proposed system. Lastly, section 5 gives some conclusions and suggestions for future work.

\section{PANORAMA SYSTEM OVERVIEW}

This section presents the panorama system outline for X-ray medical images. The input is several small sized $\mathrm{X}$-ray images and the output is one large $\mathrm{X}$-ray panorama image. The panorama system separates broadly into two stages, which are $\mathrm{x}$-ray image pre-processing and image stitching.

In the first $\mathrm{x}$-ray image pre-processing stage, the system converts a DICOM format image to a general format image, and then applies MSR to the converted image to enhance it. In the second image stitching stage, the system stitches enhanced sev- eral images and makes one large panoramic image. For that stitching process, after the system $\mathrm{ex}^{-}$ tracts the SURF points and finds the correspondences by KNN(K Nearest Neighborhood), RANSAC is used to correct the outlier and calculates the homography matrix. And then multi-band blending is invoked to fuse the images.

The proposed method is as follows.

(1) Input a set of DICOM format X-ray images

(2) Convert DICOM format images into BMP images.

(3) Enhance the converted $\mathrm{X}$-ray images based on MSR.

(4) Extract SURF from all images.

(5) Find the $\mathrm{k}$ nearest-neighborhoods for each feature using a $\mathrm{k}-\mathrm{d}$ tree data structure.

(6) From all the images, find $m$ candidate images with the most feature correspondences to the current image.

(7) Correct the outlier using RANSAC and find the homograph relation between the pairs of images.

(8) Attach all the processed images using multi-band blending.

(9) Get an enhanced panoramic image(s).

In the following sections, we will state the process above in detail.

\section{PANORAMA SYSTEM USING MSR AND SURF}

This section describes the pre-processing and stitching of $\mathrm{X}$-ray images for the proposed system. The proposed system is used for medical diagnosis in poor medical and internet environments, we are focusing on how to improve $\mathrm{X}$-ray image quality and processing speed.

\subsection{Pre-processing of $X$-ray Images}

In many cases, the qualities of $\mathrm{X}$-ray images are 
poor because images are taken using low radiation dosages for the patients' safety. The standard format of medical images is DICOM for convenient image transfer. The pre-processing consists of a process of converting the DICOM format and enhancing the poor $\mathrm{x}$-ray images by MSR.

\subsubsection{Converting DICOM Files}

In order to process the images directly on to a personal computer, they needs to be converted from the DICOM format to a general image format such as BMP [9].

A DICOM file is composed of a DICOM file header and data elements. The DICOM data element consists of a Tag, a VR (Value Representation), a VL (Value Length) and a value.

For converting the DICOM to a BMP, first, one needs to define and initialize the variables of the image parameters. Second, extract transfer syntax from Tag (0002 0010) and determine the method of reading the consequent data according to the syntax. Third, write the necessary data into related variables. Lastly, check the bit of pixels, if it is 8 , the conversion process succeeds, if not, it converts the images into 0-255 gray scale.

\subsubsection{Enhancing X-ray Images by MSR}

MSR is one of the traditional algorithms to enhance image contrast [5]. It originates from a theory on human color constancy. In our pre-experiments, it shows a good enhancement of performance for $\mathrm{X}$-ray images as in Fig. 1.

MSR is a weighted sum, which is described as:

$$
\begin{aligned}
R_{M i}(x, y)= & \sum_{n=1}^{N} W_{n} \times \log I_{i}(x, y)- \\
& \log \left[F(x, y) * I_{i}(x, y)\right]
\end{aligned}
$$

Where $\mathrm{N}$ is the number of scale, $\mathrm{F}(\mathrm{x}, \mathrm{y})$ is Gaussian kernel used to convolute with $\mathrm{I}_{\mathrm{i}}(\mathrm{x}, \mathrm{y}$ ), $\mathrm{W}_{\mathrm{n}}$ is the weighting factor and it ranges from 0 to 1 [10].

In this experiment, $\mathrm{N}=3$ is applied and for differ- ent three loops, different Gaussian kernel scale values $(\delta=54,10.3,167)$ are used.

We got the image (Fig. 1. (b)) by enhancing the original image (Fig. 1. (a)).

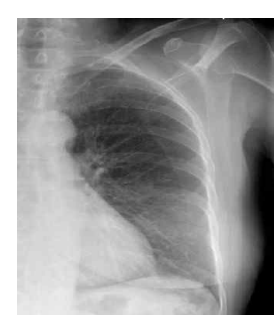

(a) Original $\mathrm{X}$-ray image

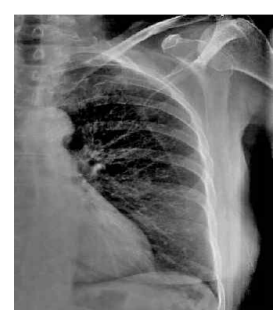

(b) Enhanced X-ray image
Fig. 1. Original and enhanced $X$-ray images based on MSR.

\subsection{Stitching X-ray Images}

This section will describe the main parts of the proposed panorama system for $\mathrm{x}$-ray images, which are image matching and image blending. Doing a simple experiment about two standard matching methods SIFT and SURF, we choose SURF because it is faster than SIFT.

\subsubsection{Overview of SIFT and SURF}

Lowe proposed SIFT in 2004 because of its invariance to image scale and rotation. It has been widely used for matching the different images of an object or scene [7]. The SIFT algorithm is based on the generation of a large collection of feature vectors and a resampling of them. Low contrast points and edge response points along the edge have been deleted. Difference-of-Gaussian function was used in the SIFT process to identify the potential interest points.

The SIFT algorithm consists of four steps, which are scale-space extreme detection, keypoint localization, orientation assignment, and keypoint descriptors.

SURF is another famous scale and rotation invariant feature point detection algorithm based on integral images, which enhanced the speed greatly, compared with SIFT the method [8]. Furthermore 
SURF preserves the property of scale-invariance based on Hessian-Laplacian to improve the speed of calculation.

SURF Descriptor detection is as follows:

(1) Calculate the integral image representation (pixel sums over upright rectangular areas) of supplied input image.

(2) Find hessian based interest points/region of integral image by doing a non maximal suppression and interpolating detection points.

(3) Extract descriptor components of interest points by calculating the dominant orientation of interest points and extracting the descriptor vector.

\subsubsection{Comparison of SIFT and SURF}

This part compares the experiments of the two methods based on output image quality and repeatability. For this comparison, we cut the original dental X-ray image in Fig. 2 into two sub-images in Fig. 3 with overlapping regions. Then, we stitch the sub-images to one image with SIFT and SURF respectively. Finally, we compare the resulting panoramic images which are shown in Fig. 4 and Fig. 5 with the original image, respectively,

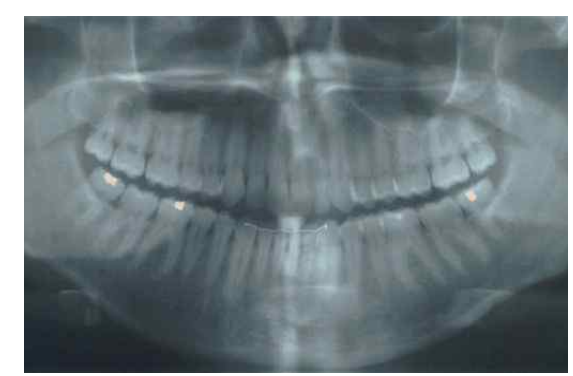

Fig. 2. Original dental $\mathrm{X}$-ray image.
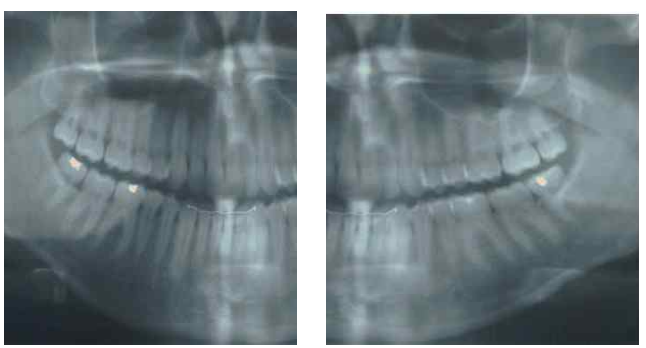

Fig. 3. Two sub-images with overlapping area.

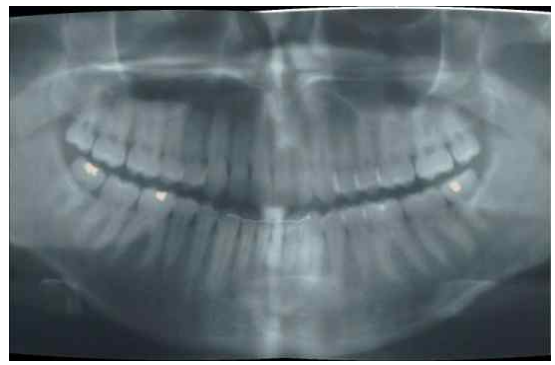

Fig. 4. Panoramic image got by SIFT.

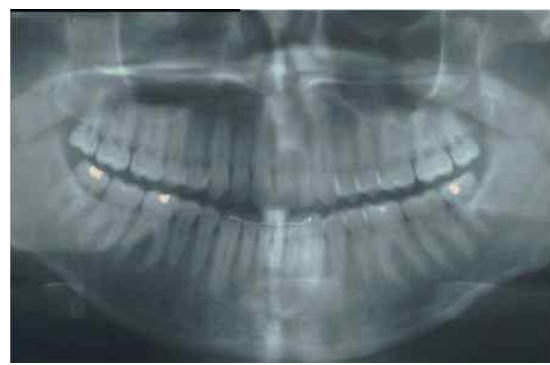

Fig. 5. Panoramic image got by SURF.

by the average gray value error equation (2).

$$
A v E r r=\frac{\sum_{i=1}^{n} \sum_{j=1}^{m}|I(i, j)-J(i, j)|}{n \times m}
$$

Where $\mathrm{nxm}$ stands for the size of the input image, and in this paper, the size of the output panoramic images was adjusted to the original image size $(509 \times 339) . I(i, j)$ is the gray value of panoramic images obtained by SIFT or SURF at point $(i, j)$ while $J(i, j)$ is the original image.

Table 1 shows the average gray value error of two panoramas to the original image. The table shows that the panoramic image acquired by SURF is more similar to the original image than by SIFT.

Table 1. Average gray value Error of SURF and SIFT

\begin{tabular}{|c|c|c|}
\hline Items & SURF & SIFT \\
\hline \hline$A v E r r^{*}$ & 1.685 & 2.269 \\
\hline
\end{tabular}

*: average gray value error

Another comparison of SIFT and SURF is carried on by the repeatability criterion, which is $\mathrm{de}^{-}$ 
fined on paper [11]:

$$
r_{1,2}=\frac{C\left(I_{1}, I_{2}\right)}{\operatorname{mean}\left(m_{1}, m_{2}\right)}
$$

Where $\mathrm{C}\left(\mathrm{I}_{1}, \mathrm{I}_{2}\right)$ stands for the number of corresponding couples, $\mathrm{m}_{1}$ and $\mathrm{m}_{2}$ denote the numbers of the detector. Table 2 shows the repeatability and time cost. We can conclude that the two methods have a similar repeatability while SURF is faster than SIFT.

Comparing SIFT with SURF on image quality, repeatability, and time cost, we can say that SURF is better than SIFT. So the proposed system adapts SURF as the feature detection algorithm.

Table 2. Repeatability and time cost of SIFT and SURF

\begin{tabular}{|c|c|c|}
\hline Items & SIFT & SURF \\
\hline \hline $\mathrm{m}^{*}$ & 90 & 30 \\
\hline $\mathrm{m} 2^{*}$ & 94 & 26 \\
\hline $\mathrm{C}\left(\mathrm{I}_{1}, \mathrm{I}_{2}\right)$ & 27 & 8 \\
\hline $\mathrm{r}_{1,2}{ }^{\$}$ & 0.293 & 0.286 \\
\hline Time Cost $(\mathrm{s})$ & 2.47 & 1.08 \\
\hline
\end{tabular}

*: number of key points, !' number of matched key points, $\$$ : repeatability

\subsection{IMAGE MATCHING}

Image matching is based on KNN and RANSAC. In $\mathrm{KNN}$, the object is classified by a majority vote of its neighbor. RANSAC attains its task by iteratively selecting a random subset of the input data. During the matching process, KNN is first applied to find the nearest 2 neighbors, and RANSAC [12] is applied to estimate a model for finding the minimal error match set. Comparing the distance of the closest neighbor with the second-closest neighbor, if it is less than the distance ratio $(r=0.5)$, the matching point is maintained, or else it is removed [7].

After the original images (a), (b), (c) shown in Fig. 11 are enhanced, they are matched with RANSAC. The matching results are shown in Fig. 6. Table 3 describes all the SURF key points and matched points of the enhanced input image.

Table 3. SURF key-points and matched points of enhanced images of Fig. 11.

\begin{tabular}{|c|c|c|}
\hline $\begin{array}{c}\text { Enhanced } \\
\text { Images }\end{array}$ & $\begin{array}{c}\text { SURF } \\
\text { Key-points }\end{array}$ & $\begin{array}{c}\text { Matched } \\
\text { points }\end{array}$ \\
\hline \hline$\left(\mathrm{a}^{\prime}\right)^{*}$ & 429 & \multirow{2}{*}{$\begin{array}{c}26\left(\left(\mathrm{a}^{\prime}\right)-\left(\mathrm{b}^{\prime}\right)\right) \\
84\left(\left(\mathrm{~b}^{\prime}\right)-\left(\mathrm{c}^{\prime}\right)\right)\end{array}$} \\
\hline$\left(\mathrm{b}^{\prime}\right)^{*}$ & 383 & \\
\hline$\left(\mathrm{c}^{\prime}\right)^{*}$ & 308 & \\
\hline
\end{tabular}

*: (a'), (b'), and (c') are enhanced images of Fig.11 (a), (b), and (c).
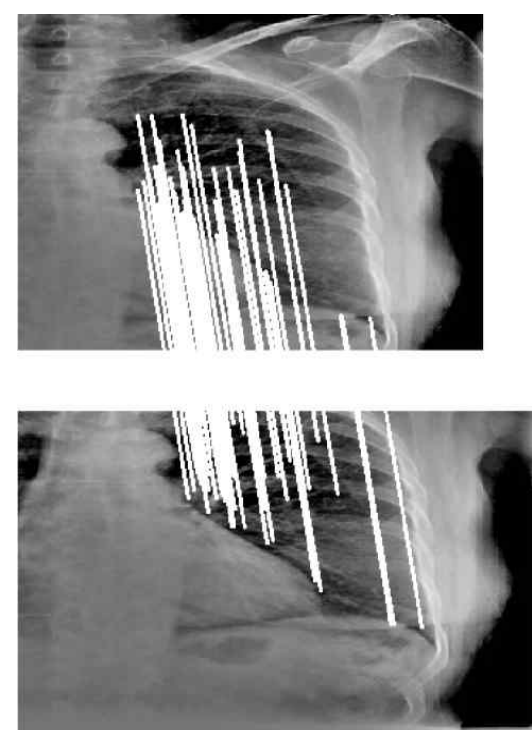

Fig. 6. Matched points of enhanced images of Fig. 11(b) and (c) by SURF.

\subsection{Image Blending}

The purpose of blending is to produce a seamless panoramic image. But because the brightness of the input images is different for each, a blending algorithm strong to bright deviations is required. In this paper, Burt's [13] multi-band blending method (2-band) which has proved effective particularly for image stitching without blurring and ghosting artifacts, has been chosen.

The point of multi-band blending is to blend low frequencies over a large spatial range, while projecting high frequencies over a short range. The image is decomposed into a series of $\mathrm{N}$ band pass 
images by Laplacian pyramid. Gaussian pyramid can be made by getting the reduced image $g_{i+1}$ from the image $g_{i}$ through the low-pass filter sequentially. Laplacian pyramid is a sequence of error image $\mathrm{L}_{0} \mathrm{~L}_{1} \cdots, \mathrm{L}_{\mathrm{n}}$. Each is the difference between two levels of the Gaussian pyramid.

The process of multi-band blending is as follows.

(1) Build Laplacian pyramid decomposition $\mathrm{X}_{1, \mathrm{k}}$ and $X_{2, k}$ for images $I_{1}$ and $I_{2}$ respectively. $X_{1, k}$ and $\mathrm{X}_{2, \mathrm{k}}$ mean the $\mathrm{k}^{\text {th }}$ level of Laplacian pyramid $\mathrm{de}^{-}$ composition respectively.

(2) Build the image mask of Gaussian pyramid decomposition: $\mathrm{M}_{\mathrm{k}}$. $\mathrm{M}_{\mathrm{k}}$ means the image mask's $\mathrm{k}^{\text {th }}$ level of Gaussian pyramid decomposition.

(3) Make a combined final image pyramid $Y_{k}$ from $X_{1, \mathrm{k}}$ and $\mathrm{X}_{2, \mathrm{k}}$ using the following equation with mask $\mathrm{M}_{\mathrm{k}}$.

$$
\begin{aligned}
Y_{k}(i, j)= & X_{1, k}(i, j) * M_{k}(i, j)+X_{2, k}(i, j) * \\
& \left(1-M_{h}(i, j)\right)
\end{aligned}
$$

Where $\mathrm{X}_{1, \mathrm{k}}$ and $\mathrm{X}_{2, \mathrm{k}}$ are the $\mathrm{k}^{\text {th }}$ level of Laplacian decomposition for the two images after coordinate adjustment, $\mathrm{Y}_{\mathrm{k}}$ is the $\mathrm{k}^{\text {th }}$ level of Laplacian pyramid decomposition for the final combination results.

More details are described in paper [6-13].

\section{EXPERIMENTAL RESULTS}

Fig. 7 and Fig. 8 show the panoramic image of the proposed system and SIFT based system with-

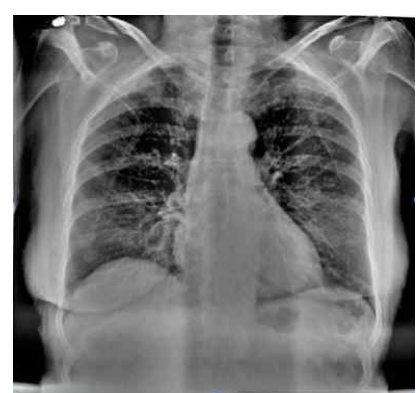

Fig. 7. Proposed system: panoramic image with 3 input images (Fig. 11) based on SURF and MSR.

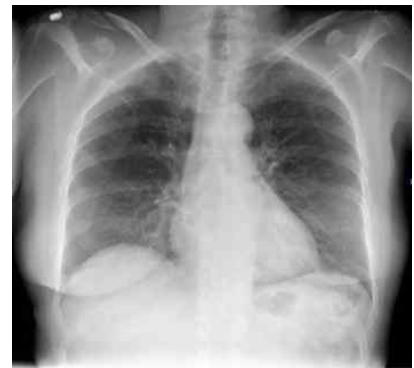

Fig. 8. Compared system: panoramic image with 3 input images (Fig. 11) based on SIFT without image enhancement.

out enhancement [2] with 3 input images (APPENDIX, Fig. 11). In order to show more input cases, Fig. 9 and Fig. 10 show the panoramic images of the proposed system and SIFT based system with 6 input images(APPENDIX, Fig. 12). They show that the panoramic image of the proposed system (Fig. 7, Fig. 9) is clearer than that of SIFT based system (Fig. 8, Fig. 10). In the proposed system, the image contrast is increased and the small details of high frequency are dis-

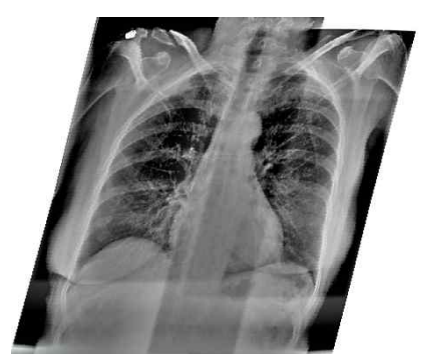

Fig. 9. Proposed system: panoramic image with 6 input images (Fig. 12) based on SURF and MSR.

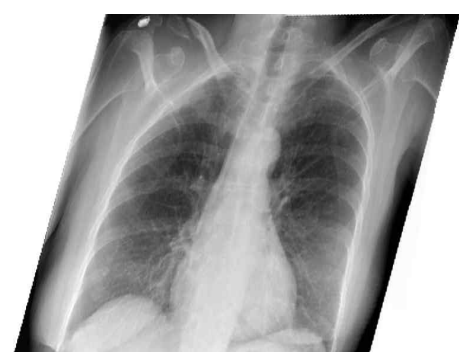

Fig. 10. Compared system: panoramic image with 6 input images (Fig. 12) based on SIFT without image enhancement. 
tinguished because the proposed system takes advantage of MSR. They show that the proposed stitching system is applicable to various numbers of input images too.

Table 4 and Table 5 give the processing time comparison of two panoramic systems with 3 input images (APPENDIX, Fig. 11) and 6 input images (APPENDIX, Fig. 12) respectively. They show that the processing time of the proposed system is almost the same as the processing time of the SIFT based system though MSR image enhancement processing is included in the proposed system.

The traditional $\mathrm{X}$-ray image panorama system is performed with a special purpose algorithm. Also it is closely combined with an image acquisition method of the application area. For example, four metal balls cast round shadows into images in a dental $\mathrm{x}$-ray image panorama system [1]. These shadows were searched and the distances acquired $\mathrm{X}$-ray stitching was done based on the information. But the proposed method is general, which has no special marker on the $\mathrm{X}$-ray image. The method can be used in any $\mathrm{X}$-ray image stitching, with no relation to the image acquisition method.

Table 4. Processing time (3 input images) of proposed system and compared system

\begin{tabular}{|c|c|c|c|c|}
\hline System & $\begin{array}{c}\text { DICOM } \\
\text { Convert } \\
(\mathrm{s})\end{array}$ & $\begin{array}{c}\text { MSR } \\
(\mathrm{s})\end{array}$ & $\begin{array}{c}\text { Image } \\
\text { Stitching } \\
(\mathrm{s})\end{array}$ & $\begin{array}{c}\text { Total } \\
(\mathrm{s})\end{array}$ \\
\hline \hline $\begin{array}{c}\text { Proposed } \\
\text { System }\end{array}$ & 7.22 & 10.11 & $\begin{array}{c}\text { (SURF) } \\
7.62\end{array}$ & 24.95 \\
\hline $\begin{array}{c}\text { Compared } \\
\text { System }\end{array}$ & 7.22 & 0 & $\begin{array}{c}\text { (SIFT) } \\
18.36\end{array}$ & 25.58 \\
\hline
\end{tabular}

Table 5. Processing time (6 input images) of proposed system and compared system

\begin{tabular}{|c|c|c|c|c|}
\hline System & $\begin{array}{c}\text { DICOM } \\
\text { Convert } \\
(\mathrm{s})\end{array}$ & $\begin{array}{c}\text { MSR } \\
(\mathrm{s})\end{array}$ & $\begin{array}{c}\text { Image } \\
\text { Stitching } \\
(\mathrm{s})\end{array}$ & $\begin{array}{c}\text { Total } \\
(\mathrm{s})\end{array}$ \\
\hline \hline $\begin{array}{c}\text { Proposed } \\
\text { System }\end{array}$ & 10.01 & 15.09 & $\begin{array}{c}\text { (SURF) } \\
9.97\end{array}$ & 35.07 \\
\hline $\begin{array}{c}\text { Compared } \\
\text { System }\end{array}$ & 10.01 & 0 & $\begin{array}{c}\text { (SIFT) } \\
24.69\end{array}$ & 34.7 \\
\hline
\end{tabular}

\section{CONCLUSION}

This paper proposed an X-ray image panorama system. The experiment shows that the system can make a clear, large, panoramic X-ray image from several small size poor quality images and the processing time of the proposed system is almost the same as the SIFT based system. Quantitatively, the average gray value error of the proposed system, which shows an image quality of 1.685 and that of a SIFT based system of 2.269. The processing time of the proposed system is $35.07 \mathrm{sec}$ and that of SIFT based system is $34.7 \mathrm{sec}$ for 6 input images each.

As the application of networks becomes widespread, developing a remote medical consultation system through the network is required. The proposed system can meet the requirements of the $\mathrm{re}^{-}$ mote image based diagnosis and treatment systems on condition that medical image transfer operations through web are included.

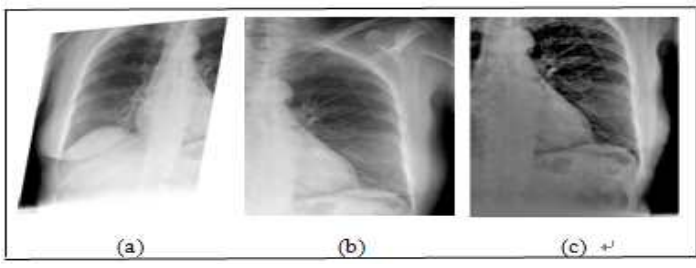

Fig. 11. Original 3 input images.

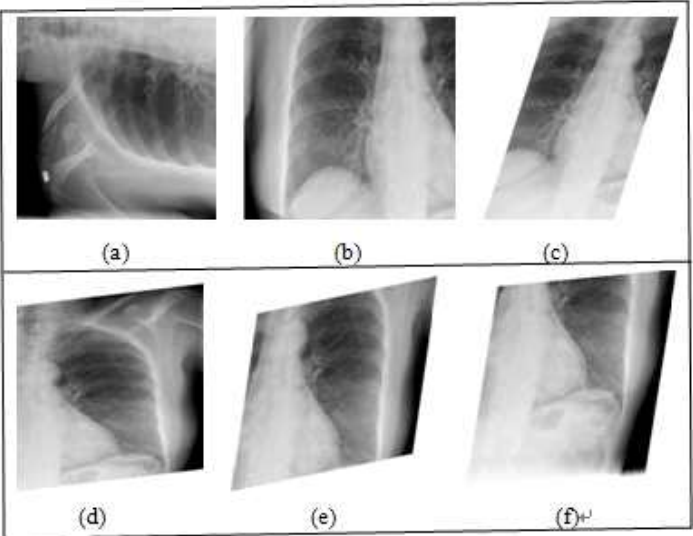

Fig. 12. Original 6 input images. 


\section{APPENDIX}

The image Fig.11 and Fig.12 above show the original input images used in this experiment.

\section{REFERENCES}

[1] T. Makela, Dental X-ray Image Stitching Algorithm, Master's Thesis of Helsinki University of Technology, 2009.

[2] Y. Ma, "A New Method of Microscopic Images' Automatic Mosaicking," Bioinformatics and Biomedical Engineering, ICBBE 2009, pp. 1-5, 2009

[3] J. Xu, "A Medical Image Mosaicking Technology Based on Venous Diagnosis," Biomedical Engineering and Computer Science, ICBECS2010, pp. 1-4, 2010.

[4] G. Lou and Y. Han, "Research on Processing and Analysis Method for Medical CT Image," 2010 International Conference on Computer and Communication Technologies in Agriculture Engineering, pp. 97-100, 2010.

[5] Z. Rahman, "Multi-Scale Retinex for Color Image Enhancement," Image Processing, Vol.3 pp. 1003-1006, 1996.

[6] M. Brown and D.G. Lowe, "Automatic Panoramic Image Stitching using Invariant Features," International Journal of Computer Vision, Vol.74, No.1, pp. 59-73, 2007.

[7] D.G. Lowe, "Distinctive Image Features from Scale Invariant Keypoints," International Journal of Computer Vision, Vol.60, No.2, pp. 91-110, 2004.

[8] H. Bay, A. Ess, T. Tuytelaars, and L. V. Gool, "SURF: Speeded Up Robust Features," Computer Vision and Image Understanding, Vol.110, No.3, pp. 346-359, 2008.

[9] G. Lou and Y. Han, "Research on Processing and Analysis Method for Medical CT Image," 2010 International Conference on Computer and Communication Technologies in Agriculture Engineering, pp. 97-100, 2010
[10] D.J. Jobson and Z. Rahman, "Properties and Performance of a Center/Surround Retinex," IEEE Trans. on Image Processing, Vol.6, No.3, pp. 451-462, 1997.

[11] J. Lou and O. Gwun, "A Comparison of SIFT, PCA-SIFT and SURF," International Journal of Image Processing, Vol.3, No.4, pp. 143-152, 2009.

[12] M.A. Fischler and R.C. Bolles, "Random Sample Consensus: A Paradigm for Model Fitting with Applications to Image Analysis and Automated Cartography," Comm. of the ACM, Vol.24, No. 6, pp. 381-395, 1981.

[13] P.J. Burt and E.H. Adelson, "A Multiresolution Spline with Application to Image Mosaics," ACM Trans. On Graphics, Vol.2, No.4, pp. 217-236, 1983.

[14] L. Juan, S. Shin, H. Park, and O. Gwun, "Stitching for Panorama Based on SURF and Multi-band Blending," Journal of Korea Multimedia Society, Vol.14, No.2, pp.201-209, 2011.

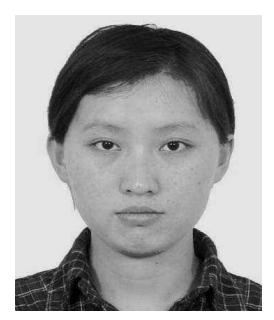

Weiwei Wang

2009. 7: Division of Computer Sci. \& Eng., South Central University for Nationalities (B.S.)

2012. 2: Division of Computer Sci. \& Eng., Chonbuk National University (M.S.) Research interests: Computer Graphics, Medical Image Processing

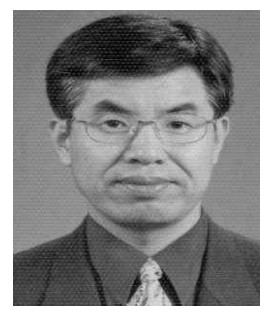

Oubong Gwun

1980. 2: Electrical Eng., Korea University (B. S.)

1983. 8: Electrical Eng., Korea University (M. S)

1993. 3: Interdisciplinary Graduate School of Eng. Sci., Kyushu University (Ph. D)

1992. 4 1993. 9: Assistant prof., Dept. of Computer Eng., Kyushu University

1993. 10 Present: Prof., Division of Computer Sci. \& Eng., Chonbuk National University.

Research interest: Computer Graphics, Visualization 